\title{
A New Current Conveyor Full-Wave Rectifier for Low Frequency/Small Signal Medical Applications
}

\author{
Adisak Monpapassorn \\ Department of Electrical Engineering, Southeast Asia University, Bangkok, Thailand \\ Email: aone_mon@hotmail.com
}

How to cite this paper: Monpapassorn, A. (2018) A New Current Conveyor Full-Wave Rectifier for Low Frequency/Small Signal Medical Applications. Circuits and Systems, 9, 58-65.

https://doi.org/10.4236/cs.2018.93006

Received: February 26, 2018

Accepted: March 20, 2018

Published: March 23, 2018

Copyright $\odot 2018$ by author and Scientific Research Publishing Inc. This work is licensed under the Creative Commons Attribution International License (CC BY 4.0).

http://creativecommons.org/licenses/by/4.0/

\section{(c) (i) Open Access}

\begin{abstract}
This paper presents a new current conveyor (CCII+) full-wave rectifier for low frequency/small signal medical applications. The proposed rectifier is based on the current conveyor full-wave rectifier proposed previously, but the proposed rectifier is better in view of no need diodes to rectify, and no need bias sources to overcome the zero crossing error. It needs only two CCII $+\mathrm{s}$, two resistors, and three simple current mirrors, which is easy for IC implementation and for building in many countries. The PSPICE simulation with the current conveyor CCII+ in the current feedback opamp AD844 IC and the $2 \mathrm{~N} 2222$ bipolar current mirror shows the good low frequency/small signal rectification, the operation voltage of down to $6 \mu \mathrm{V}$.
\end{abstract}

\section{Keywords}

Full-Wave Rectifier, Current Conveyor, Low Frequency, Medical Circuit

\section{Introduction}

In the past few decades, medical and biological researches gained more and more attention. The ever-growing need for such researches caused many medical applications to develop. Collecting data from a living human body is a complex task and needs circumspection.

Low frequency full-wave rectifiers are important building blocks for small signal processing in biomedical systems. These circuits should not introduce any form of distortion that can destroy the information contained. For this reason, the analog processing blocks must present high performance over the frequency of interest.

As well-known the operation of diode-only rectifier is limited by the threshold 
voltage of the diode, approximately $0.3 \mathrm{~V}$ for the germanium diode and $0.7 \mathrm{~V}$ for the silicon diode. Thus, the diode-only rectifier is used in those applications in which the precision in the range of threshold voltage is insignificant. For application requiring high accuracy, the diode-only rectifiers cannot be used; integrated circuit (IC) rectifiers are used instead.

One advantage of the IC rectifier designed using the simple devices such as opamps, current conveyors (CCII), current mirrors, diodes, and resistors as components is that they can be built in many countries.

Recently, the current mode full-wave rectifier using dual CCII as the voltage to current converter has received much attention. For example, [1] and [2] proposed the same dual CCII full-wave rectifier as shown in Figure 1(a). This full-wave rectifier is developed to reduce the distortion due to the small-signal $\mathrm{dV} / \mathrm{dt}$ limitation at the next time by [3] with the addition of a DC voltage source as shown in Figure 1(b). [4] further developed this rectifier to reduce the effect of temperature on the zero crossing performance by using a current source and a resistor in place of the voltage source as shown in Figure 1(c). In 2013, the author [5] proposed the dual CCII full-wave rectifier with low output impedance advantage by redesigning the circuit as shown in Figure 2.

In this paper, the author presents a new CCII full-wave rectifier for low frequency/small signal medical applications that is better than the previous CCII full-wave rectifiers as follows.

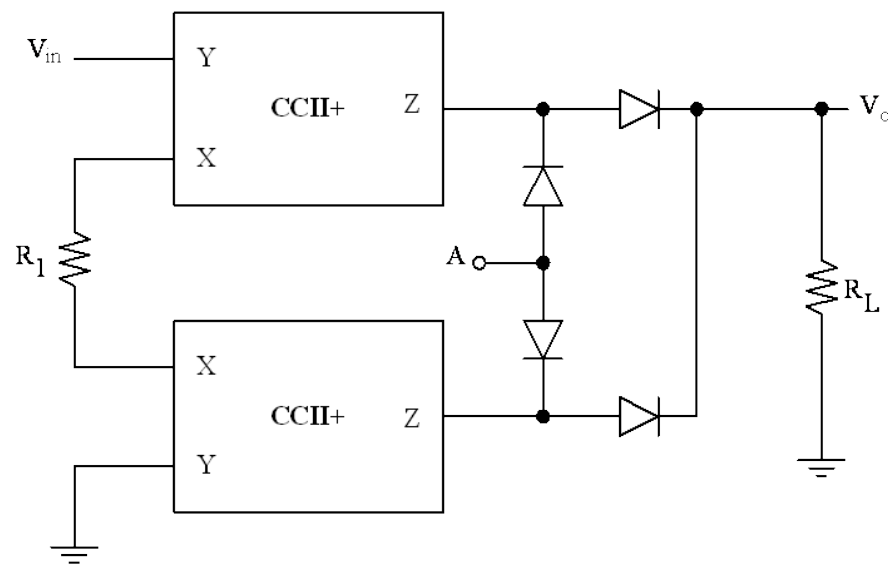

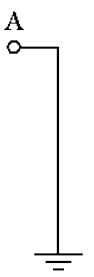

(a)

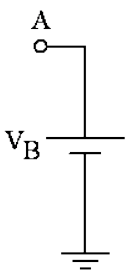

(b)

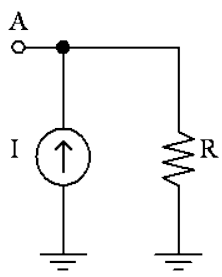

(c)

Figure 1. CCII based precision full-wave rectifiers: (a) proposed by [1] and [2], (b) voltage biasing technique proposed by [3], and (c) current biasing technique proposed by [4]. 


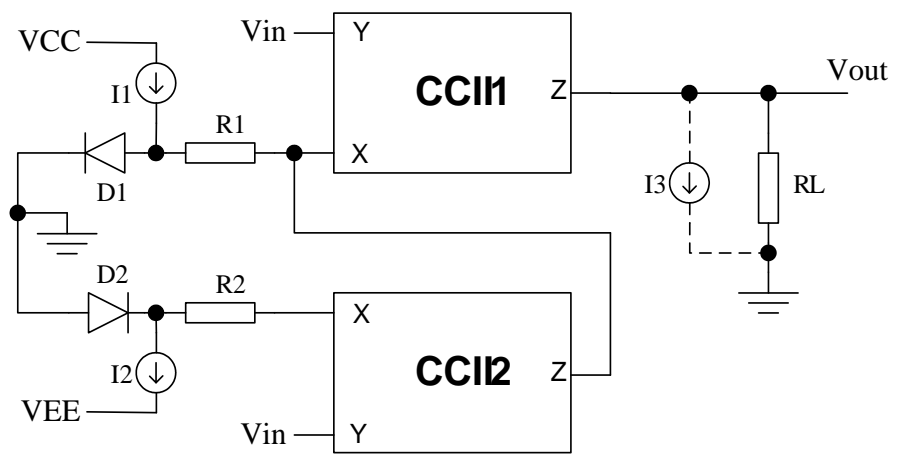

Figure 2. Low output impedance version proposed by [5].

- The proposed rectifier does not consist of the diodes as the previous rectifiers [1] [2] [3] [4] [5]. It requires one solid state device, only bipolar or MOS transistors for CCIIs and current mirrors. Thus the proposed rectifier is easier in IC fabrication.

- The proposed rectifier does not use the bias sources to make diodes turning-on all the time to overcome the zero crossing error as the previous rectifiers [3] [4] [5]. Its rectifier core (current mirrors) operates as a push-pull mode; with this push-pull operation the zero crossing error can be canceled.

- The proposed rectifier has the temperature stability on the zero crossing performance to be better than the previous rectifiers [1] [2] [3] [4] [5].

\section{Proposed CCII Full-Wave Rectifier}

The proposed full-wave rectifier is shown in Figure 3. The rectifier consists of two CCII+s, two resistors, and three simple bipolar current mirrors. If the solid-state resistor is used, the proposed full-wave rectifier can be build by using only one kind of devices, i.e. MOS or bipolar transistors for CCII $+\mathrm{s}$, current mirrors and solid-state resistors. It is so easy for the IC process.

The operation of the circuit is as follows. The second generation current conveyor CCII+ [6], the relation of the voltage and current at nodes $\mathrm{X}, \mathrm{Y}$ and $\mathrm{Z}$ can be expressed as

$$
\left.\begin{array}{c}
i_{Y}=0 \\
v_{X}=v_{Y} \\
i_{Z}=i_{X}
\end{array}\right\}
$$

Figure 3 and Equation (1) show that, the input voltage is fed to nodes $Y$ of CCII1 and CCII 2 and thus follows to their nodes $X$. The voltage between nodes $X$ of two CCIIs makes the input current $I_{i n}$ through $R_{i n}$. This input current is mirrored to nodes $Z$ of two CCIIs as the two out-of-phase currents.

$$
i_{Z 1}=-i_{Z 2}=\frac{V_{i n}}{R_{i n}}=I_{\text {in }}
$$

where the input voltage $V_{i n}$ is $\left(V_{i n t}-V_{i n-}\right)$.

When $V_{i n+}$ is positive, $V_{i n-}$ is negative, the node $Z$ currents of CCII1 and CCII2 make the current mirrors [7] CM1 to operate and CM2 to not operate. 


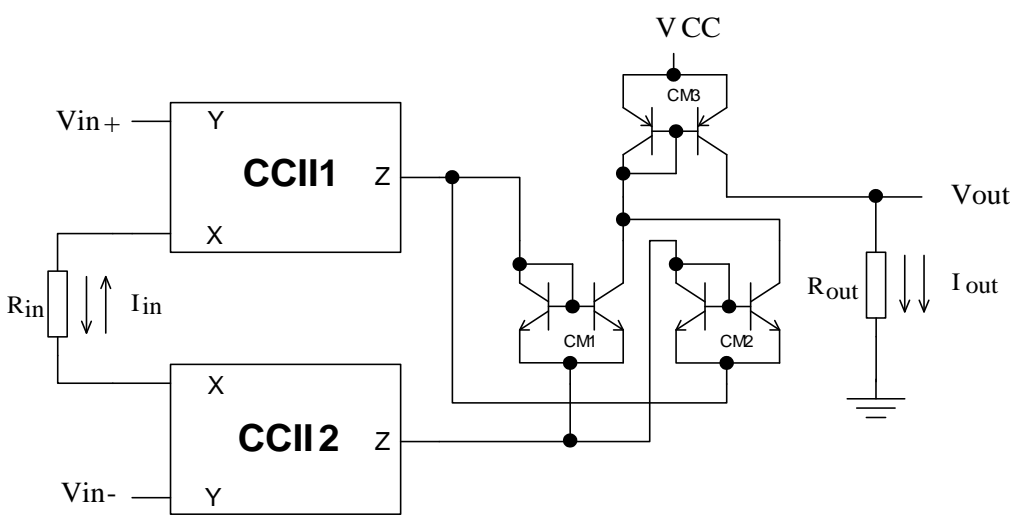

Figure 3. Proposed CCII full-wave rectifier.

The current at the output of CM1 $\left(I_{i n}\right)$ is the negative current (the current flows into the output of CM1).

When $V_{i n+}$ is negative, $V_{i n-}$ is positive, the node $Z$ currents of CCII1 and CCII2 make the current mirrors CM2 to operate and CM1 to not operate. The current at the output of CM2 $\left(I_{i n}\right)$ is also the negative current (the current flows into the output of CM2).

Note that, when $V_{i n}$ is positive the output current of CM1 is $-I_{\text {in }}$ and one of CM2 is zero, and when $V_{\text {in }}$ is negative the output current of CM2 is $I_{\text {in }}$ and one of CM1 is zero. Both output currents of the current mirrors (CM1 and CM2) are added and mirrored by CM3 to convert the direction of currents as the output of CM3. This output current is changed to the output voltage by $R_{\text {out }}$ The relation between the input voltage and the output voltage of the proposed full-wave rectifier can be written as

$$
\left.\begin{array}{rl}
V_{\text {in }}>0 & \Rightarrow V_{\text {out }}=A_{V} V_{\text {in }} \\
V_{\text {in }}<0 & \Rightarrow V_{\text {out }}=-A_{V} V_{\text {in }}
\end{array}\right\}
$$

Because of the two out-of-phase currents at the outputs of two CCIIs, the rectifier cores (CM1 and CM2) are driven in the push-pull mode; hence, the zero crossing error can be canceled. The proposed full-wave rectifier does not use the bias sources to make diodes turning-on all the time to overcome the zero crossing error as the previous full-wave rectifiers [3] [4] [5].

Using the small signal model of the CCII in Figure 4, the gain of the proposed CCII full-wave rectifier in Figure 3 can be written as

$$
A_{V}=\frac{R_{\text {out }} \alpha_{N} \alpha_{P}}{R_{\text {in }}+2 R_{x}}
$$

where $\alpha_{N}$ is the current gain of the simple NPN bipolar current mirror, $\alpha_{P}$ is the current gain of the simple PNP bipolar current mirror [7], and $R_{X}$ is the internal resistance at node $X$ of the CCII [1] [6].

A comparison between the proposed CCII full-wave rectifier and the previous CCII full-wave rectifiers [1] [2] [3] [4] [5] was done. The comparison result is shown in Table 1. This comparison is based on a single mode input as [5]. Note 

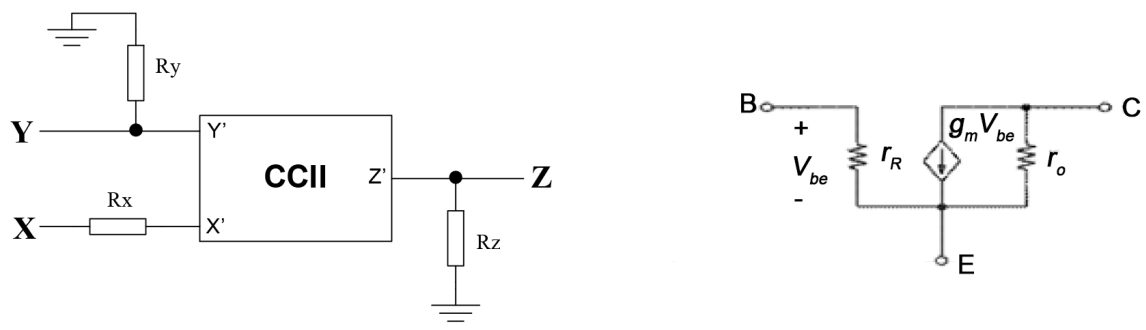

Figure 4. Low frequency small signal models: current conveyor on the left and bipolar transistor on the right.

Table 1. Comparison between the proposed CCII full-wave rectifier and the previous CCII full-wave rectifiers.

\begin{tabular}{cccc}
\hline & Proposed rectifier & CCII rectifiers [1] [2] [3] [4] & CCII rectifier [5] \\
\hline Input impedance & $R_{y}$ & $R_{Y}$ & $R_{y} / 2$ \\
Differential mode input & Yes & Yes & No \\
Output impedance & $R_{\text {out }} / / r_{o}$ & $R_{L} / /\left(r_{D(\text { on })}+R_{z}\right)$ & $R_{L} / / R_{z}$ \\
Output voltage range & Current mirror range & CCII range-diode & CCII range \\
threshold voltage & 2 \\
Diodes & - & 4 & 3 \\
\hline Resistors & 2 & 2 &
\end{tabular}

that the proposed rectifier does not use diodes as the previous rectifiers [1] [2] [3] [4] [5]. If the solid-state resistor is used, the proposed full-wave rectifier can be build by using only one kind of devices, i.e. MOS or bipolar transistors for CCII+s, current mirrors and solid-state resistors. Thus the proposed rectifier is easier in IC fabrication.

Using the small signal model in Figure 4, because of the same input structure, the input impedance of the proposed rectifier is as good as ones of [1] [2] [3] [4], with the same differential mode input advantage.

Considering the output impedance, the proposed full-wave rectifier has the low output impedance, the same as the low output impedance full-wave rectifier [5] because most of the CCIIs use the current mirror at the output stage. Also, the wide output voltage range advantages are the same between the proposed full-wave rectifier and the full-wave rectifier [5].

\section{Simulation Results}

The proposed full-wave rectifier was simulated by using the PSPICE program (OrCAD Release 9.1). The AD 844 ICs from Analog Devices (commercially CCII [2]) and 2N2222 bipolar transistors were used in simulation. The supply voltages for the circuit are $\mathrm{V}_{\mathrm{CC}}=+12 \mathrm{~V}$ and $\mathrm{V}_{\mathrm{EE}}=-12 \mathrm{~V}$. Resistors: $\mathrm{R}_{\mathrm{in}}=50 \mathrm{ohms}$ and $\mathrm{R}_{\text {out }}=230 \mathrm{ohms}$ were chosen. This resistance will make the gain of the proposed full-wave rectifier (4) about 1; considering the 50 ohms $\mathrm{R}_{\mathrm{x}}$ of the CCII in AD844 IC and the error of current mirrors. However, in the IC process, the supply voltages for the CCII can be the lower voltage if the low voltage CCII is used. 
The input sine wave signal $\left(100 \mathrm{mV}_{\text {peak }}\right)$ is fed to the input of the proposed CCII full-wave rectifier. The output signal is displayed in Figure 5(a)-(c) for the frequencies of $1 \mathrm{~Hz}, 10 \mathrm{~Hz}$ and $100 \mathrm{~Hz}$, respectively, in which is the complete full-wave waveform.

The zero-crossing performance of the proposed CCII full-wave rectifier is magnified and shown in Figure 6. The operation temperatures $\left(0^{\circ} \mathrm{C}\right.$ lower, $27^{\circ} \mathrm{C}$ middle, and $125^{\circ} \mathrm{C}$ upper) were selected; normally, these temperature errors

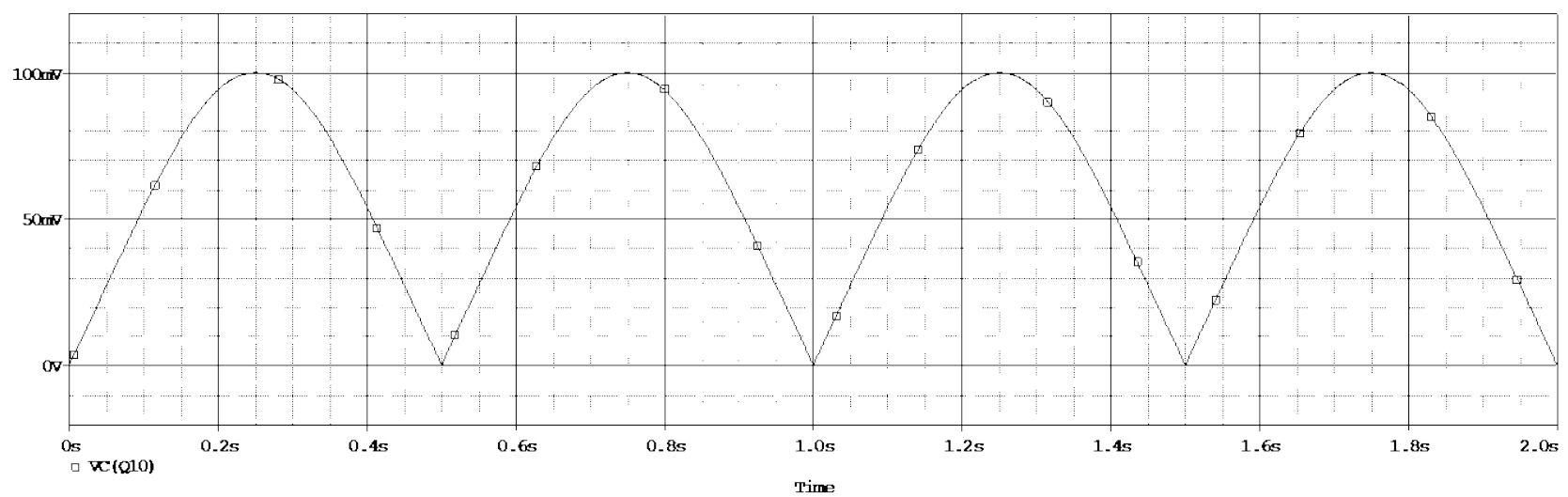

(a)

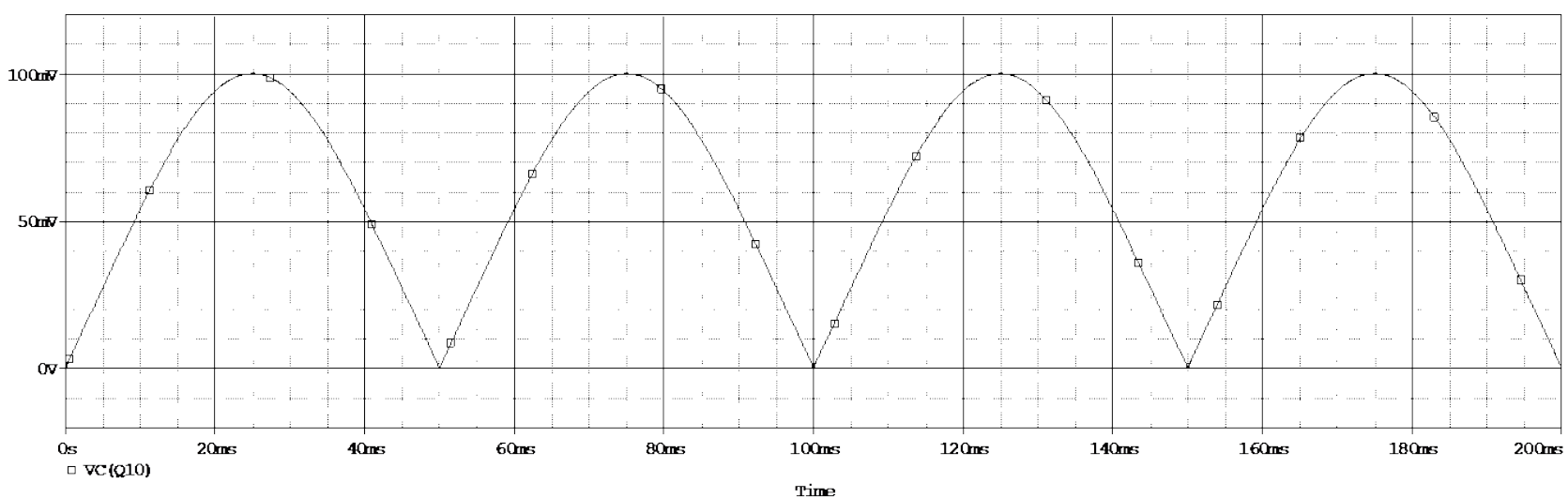

(b)

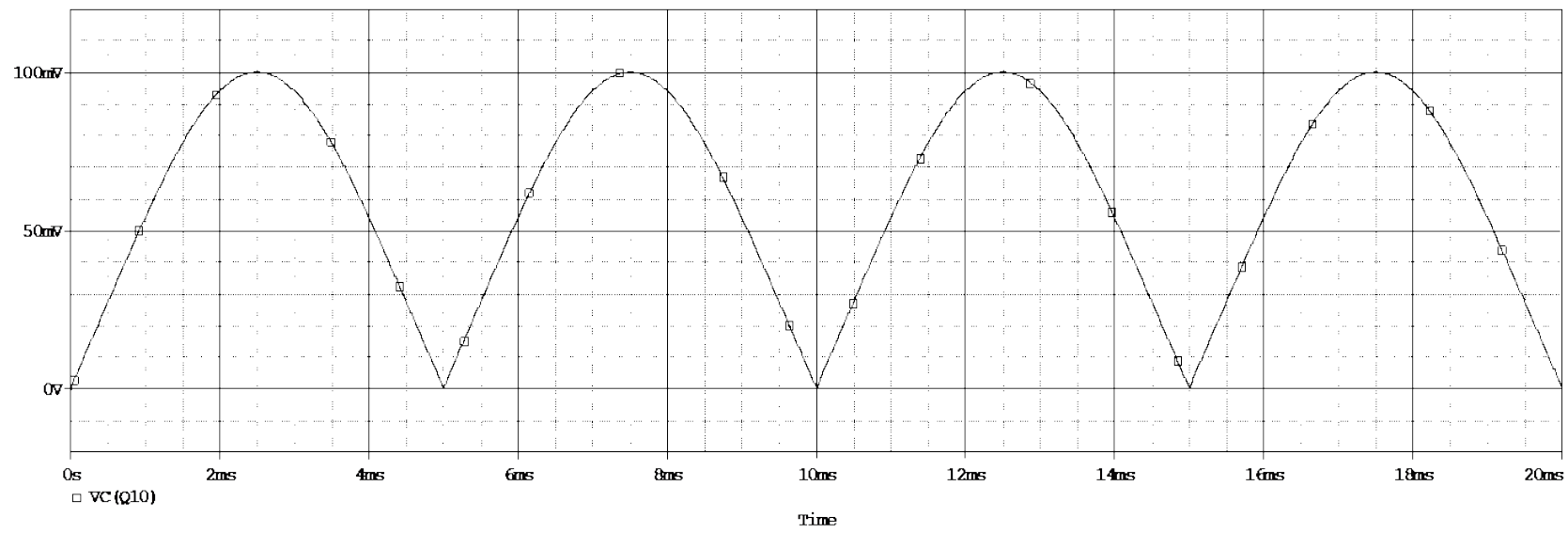

(c)

Figure 5. Output signals with: (a) $1 \mathrm{~Hz}$ sine wave input; (b) $10 \mathrm{~Hz}$ sine wave input; and (c) $100 \mathrm{~Hz}$ sine wave input. 


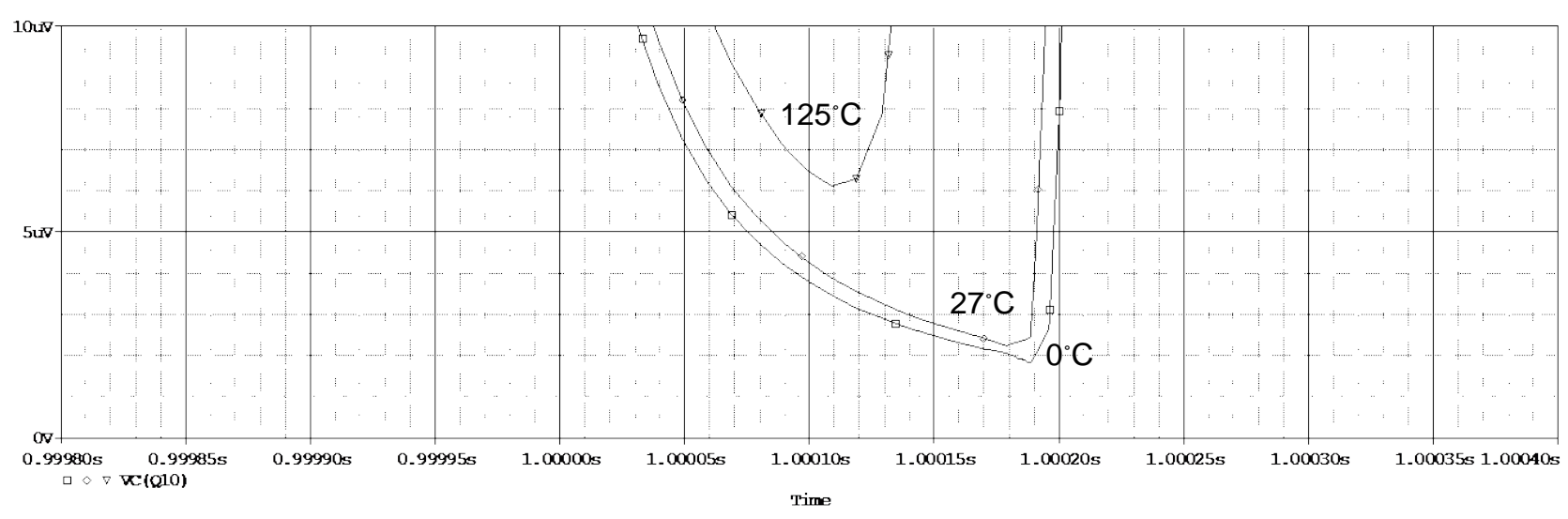

Figure 6. Zero-crossing performance of the proposed CCII full-wave rectifier.

cannot be seen with the X-Y scale of Figure 5. Figure 6 shows that the proposed CCII full-wave rectifier can rectify the minimum voltage of $6 \mu \mathrm{V}$.

Comparing the previous CCII full-wave rectifiers [1] [2] [3] [4] [5], the best zero-crossing temperature performance is of the rectifier [4] that has showed the result as follows. The minimum operation voltages at the temperatures of $27^{\circ} \mathrm{C}$, $50^{\circ} \mathrm{C}$, and $70^{\circ} \mathrm{C}$ are about $8 \mathrm{mV}, 16 \mathrm{mV}$, and $29 \mathrm{mV}$, respectively. However, for the proposed CCII full-wave rectifier, Figure 6 shows the minimum operation voltage at the temperatures of $0^{\circ} \mathrm{C}, 27^{\circ} \mathrm{C}$, and $125^{\circ} \mathrm{C}$ are about $2 \mu \mathrm{V}, 2.2 \mu \mathrm{V}$, and $6 \mu \mathrm{V}$, respectively.

It is crystal clear that the zero-crossing temperature performance of the proposed rectifier is better than those of the previous rectifiers [1] [2] [3] [4] [5]. This is because of the push-pull operation of the rectifier cores as described in the above section. But the previous full-wave rectifiers [1] [2] [3] [4] [5] operate only in the push mode.

\section{Conclusion}

In this paper, the author has reported a new CCII full-wave rectifier for low frequency/small signal medical applications, which consists of two CCIIs, two resistors, and three simple current mirrors. The proposed CCII full-wave rectifier operates in a push-pull mode. With these, the proposed rectifier yields the advantages over the previous CCII full-wave rectifiers [1] [2] [3] [4] [5] in view of the IC fabrication process, and a zero-crossing performance both the minimum operation voltage and the temperature stability. The simulation results confirm the theory and the advantage. The proposed rectifier is suitable for a high impedance external load. If the low impedance load is applied, the proposed rectifier needs a voltage buffer.

\section{References}

[1] LTP Electronics LTD (1993) CCII01 Current Conveyor Data Sheet. LTP Electronics LTD, Oxford.

[2] Khan, A.A., Abou el-ela, M. and Al-turaigi, M.A. (1995) Current Mode Precision 
Rectification. International Journal of Electronics, 79, 853-859. https://doi.org/10.1080/00207219508926319

[3] Toumazou, C., Lidgey, F.J. and Chattong, S. (1994) High Frequency Current Conveyor Precision Full-Wave Rectifier. Electronics Letters, 30, 745-746. https://doi.org/10.1049/el:19940539

[4] Hayatleh, K., Porta, S. and Lidgey, F.J. (1994) Temperature Independent Current Conveyor Precision Rectifier. Electronics Letters, 30, 2091-2093. https://doi.org/10.1049/el:19941454

[5] Monpapapassorn, A. (2013) Low Output Impedance Dual CCII Full-Wave Rectifier. International Journal of Electronics, 100, 648-654. https://doi.org/10.1080/00207217.2012.720943

[6] Sedra, A. and Smith, K.C. (1970) A Second Generation Current Conveyor and Its Applications. IEEE Transaction on Circuit Theory, 17, 132-134. https://doi.org/10.1109/TCT.1970.1083067

[7] Toumazou, C., Lidgey, F.J. and Haigh, D.G. (1990) Analogue IC Design: The Current Mode Approach. Peter Peregrinus Ltd., London. 